\title{
Dark Matter and Baryogenesis from Non-Abelian Gauged Lepton Number
}

\author{
Bartosz Fornal \\ Department of Physics, University of California, San Diego \\ 9500 Gilman Drive, La Jolla, CA 92093, USA
}

\begin{abstract}
A simple model is constructed based on the gauge symmetry $S U(3)_{c}$ $\times S U(2)_{L} \times U(1)_{Y} \times S U(2)_{\ell}$, with only the leptons transforming nontrivially under $S U(2)_{\ell}$. The extended symmetry is broken down to the Standard Model gauge group at $\mathrm{TeV}$-scale energies. We show that this model provides a mechanism for baryogenesis via leptogenesis in which the lepton number asymmetry is generated by $S U(2)_{\ell}$ instantons. The theory also contains a dark matter candidate - the $S U(2)_{\ell}$ partner of the right-handed neutrino.
\end{abstract}

\footnotetext{
*Plenary talk given at the Conference on Cosmology, Gravitational Waves and Particles, Singapore, February 6-10, 2017; based on the work: B. Fornal, Y. Shirman, T. M. P. Tait and J. R. West, arXiv:1703.00199 [hep-ph] [1].
} 


\section{Introduction}

The Standard Model of elementary particle physics provides an extremely accurate description of Nature at the most fundamental level. Despite its remarkable successes, it explains only $5 \%$ of the Universe, while the remaining $95 \%$ is attributed to the mysterious dark matter and dark energy. In addition, the Standard Model itself has its own shortcomings: the inability to generate the observable matter-antimatter asymmetry of the Universe, the hierarchy problem, massless neutrinos, unknown origin of flavor, and many more. Although a plethora of models dealing with those issues have been constructed, it is still an open question which of those theories, if any, provides the correct or at least partially correct description of Nature at higher energies. We simply need more experimental data to find this out. In the meantime, further systematizing and rethinking of our model building efforts is definitely required.

The Standard Model is based on the gauge group $S U(3)_{c} \times S U(2)_{L} \times U(1)_{Y}$ [2, 3, 4, 5, 6]. Apart from this local symmetry, it also has two accidental global symmetries: baryon number and lepton number. One might wonder whether those are just residual symmetries left over from the breaking of a more fundamental extended gauge symmetry. Efforts of gauging baryon and lepton number were carried in the past [7, 8, 9, 10, 11, 12], but only the models constructed recently [13, 14, 15, 16 are experimentally viable. In theories of this type gauge coupling unification does not occur naturally and so far only partial unification has been achieved [17, 18].

Nevertheless, simple extensions of the Standard Model gauge group provide a good playground for testing various approaches to the dark matter and baryogenesis puzzles. In this talk, I will discuss one of such extensions containing a dark matter candidate and offering a mechanism for producing a lepton asymmetry, which ultimately can explain the matter-antimatter asymmetry of the Universe.

\section{The model}

The theory we propose is based on the gauge group:

$$
S U(3)_{c} \times S U(2)_{L} \times U(1)_{Y} \times S U(2)_{\ell} .
$$




\begin{tabular}{|c||c||c|c|}
\hline Field & $S U(2)_{\ell}$ & $S U(2)_{L}$ & $U(1)_{Y}$ \\
\hline \hline$\hat{l}_{L}$ & 2 & 2 & $-\frac{1}{2}$ \\
\hline$\hat{e}_{R}$ & 2 & 1 & -1 \\
\hline$\hat{\nu}_{R}$ & 2 & 1 & 0 \\
\hline \hline$l_{R}^{\prime}$ & 1 & 2 & $-\frac{1}{2}$ \\
\hline$e_{L}^{\prime}$ & 1 & 1 & -1 \\
\hline$\nu_{L}^{\prime}$ & 1 & 1 & 0 \\
\hline \hline$\hat{\Phi}_{1,2}$ & 2 & 1 & 0 \\
\hline
\end{tabular}

Table 1: New field representations in the model.

\section{$2.1 \quad$ Fermionic sector}

The Standard Model quarks are singlets under $S U(2)_{\ell}$, whereas the lefthanded lepton doublet $l_{L}$ and the right-handed electron $e_{R}$ are the upper components of $S U(2)_{\ell}$ doublets,

$$
\hat{l}_{L}=\left(\begin{array}{c}
l \\
\tilde{l}
\end{array}\right)_{L}, \quad \hat{e}_{R}=\left(\begin{array}{c}
e \\
\tilde{e}
\end{array}\right)_{R},
$$

where the lower components are the new partner fields $\tilde{l}_{L}$ and $\tilde{e}_{R}$, respectively. To cancel the gauge anomalies involving $S U(2)_{\ell}$ one requires an extra $S U(2)_{\ell}$ doublet of Standard Model singlet fields,

$$
\hat{\nu}_{R}=\left(\begin{array}{c}
\nu \\
\tilde{\nu}
\end{array}\right)_{R} .
$$

The remaining anomalies involving just the Standard Model gauge groups are canceled by introducing new $S U(2)_{\ell}$ singlet fields:

$$
l_{R}^{\prime}, \quad e_{L}^{\prime}, \quad \nu_{L}^{\prime} .
$$

The particle content of the model along with the quantum numbers of the fields is shown in Table 1. The Standard Model quarks were not included since they transform trivially under $S U(2)_{\ell}$. 


\subsection{Higgs and gauge sector}

Although breaking of the extended gauge group down to the Standard Model can be achieved with just one new $S U(2)_{\ell}$ doublet Higgs, for reasons discussed later we introduce two new Higgs fields $\hat{\Phi}_{1,2}$ and assume that one of the vacuum expectation values (vevs) is much larger than the other, $v_{1} \gg v_{2}$. This can be easily engineered by choosing appropriate values for the parameters in the scalar potential:

$$
\begin{aligned}
V\left(\Phi_{1}, \Phi_{2}\right) & =m_{1}^{2}\left|\hat{\Phi}_{1}\right|^{2}+m_{2}^{2}\left|\hat{\Phi}_{2}\right|^{2}+\left(m_{12}^{2} \hat{\Phi}_{1}^{\dagger} \hat{\Phi}_{2}+\text { h.c. }\right) \\
& +\lambda_{1}\left|\hat{\Phi}_{1}\right|^{4}+\lambda_{2}\left|\hat{\Phi}_{2}\right|^{4}+\lambda_{3}\left|\hat{\Phi}_{1}\right|^{2}\left|\hat{\Phi}_{2}\right|^{2}+\lambda_{4}\left|\hat{\Phi}_{1}^{\dagger} \hat{\Phi}_{2}\right|^{2} \\
& +\left[\tilde{\lambda}_{5} \hat{\Phi}_{1}^{\dagger} \hat{\Phi}_{2}\left|\hat{\Phi}_{1}\right|^{2}+\tilde{\lambda}_{6} \hat{\Phi}_{1}^{\dagger} \hat{\Phi}_{2}\left|\hat{\Phi}_{2}\right|^{2}+\tilde{\lambda}_{7}\left(\hat{\Phi}_{1}^{\dagger} \hat{\Phi}_{2}\right)^{2}+\text { h.c. }\right],
\end{aligned}
$$

where we neglected terms involving the Standard Model Higgs field. After $\hat{\Phi}_{1,2}$ develop vevs,

$$
\left\langle\hat{\Phi}_{i}\right\rangle=\frac{1}{\sqrt{2}}\left(\begin{array}{c}
0 \\
v_{i}
\end{array}\right),
$$

the $S U(3)_{c} \times S U(2)_{L} \times U(1)_{Y} \times S U(2)_{\ell}$ symmetry is broken down directly to the Standard Model gauge group.

Apart from the new Higgs particles, the theory contains three new vector gauge bosons:

$$
Z^{\prime}, W_{+}^{\prime}, W_{-}^{\prime},
$$

which do not mix with the Standard Model electroweak gauge bosons.

\subsection{Particle masses}

The Yukawa part of the Lagrangian is given by:

$$
\begin{aligned}
\mathcal{L}_{\mathrm{Y}} & =\sum_{i}\left[Y_{l}^{a b} \overline{\hat{l}}_{L}^{a} \hat{\Phi}_{i} l_{R}^{b}+Y_{e}^{a b} \overline{\hat{e}}_{R}^{a} \hat{\Phi}_{i} e_{L}^{\prime b}+Y_{\nu}^{a b} \overline{\hat{\nu}}_{R}^{a} \hat{\Phi}_{i} \nu_{L}^{\prime b}\right] \\
& +y_{e}^{a b} \overline{\hat{l}}_{L}^{a} H \hat{e}_{R}^{b}+y_{\nu}^{a b} \overline{\hat{l}}_{L}^{a} \tilde{H} \hat{\nu}_{R}^{b}+y_{e}^{\prime a b} \bar{l}_{R}^{\prime a} H e_{L}^{\prime b}+y_{\nu}^{\prime a b} \bar{l}_{R}^{a} \tilde{H} \nu_{L}^{\prime b}+\text { h.c. },
\end{aligned}
$$

where $a, b=1,2,3$ are flavor indices. After $S U(2)_{\ell}$ symmetry breaking, the Yukawa matrices $Y_{l}, Y_{e}$ and $Y_{\nu}$ lead to vector-like masses for all new fermions 
in the theory. The Yukawa matrices $y_{e}$ and $y_{\nu}$ produce the usual Standard Model lepton masses and, along with $y_{e}^{\prime}$ and $y_{\nu}^{\prime}$, contribute also to lepton partner masses. The fermionic mass matrix is given by:

$$
\begin{aligned}
& \frac{1}{\sqrt{2}}\left(\begin{array}{ll}
\bar{\nu}_{L} & \bar{\nu}_{L}^{\prime}
\end{array}\right)\left(\begin{array}{cc}
Y_{l} v_{\ell} & y_{\nu} v \\
y_{\nu}^{\prime \dagger} v & Y_{\nu}^{\dagger} v_{\ell}
\end{array}\right)\left(\begin{array}{c}
\nu_{R}^{\prime} \\
\tilde{\nu}_{R}
\end{array}\right)+\frac{1}{\sqrt{2}}\left(\begin{array}{cc}
\tilde{e}_{L} & \bar{e}_{L}^{\prime}
\end{array}\right)\left(\begin{array}{cc}
Y_{l} v_{\ell} & y_{e} v \\
y_{e}^{\prime \dagger} v & Y_{e}^{\dagger} v_{\ell}
\end{array}\right)\left(\begin{array}{c}
e_{R}^{\prime} \\
\tilde{e}_{R}
\end{array}\right) \\
& + \text { h.c. }
\end{aligned}
$$

where $v_{\ell}=\sqrt{v_{1}^{2}+v_{2}^{2}}$ and $v$ is the Standard Model Higgs vev. The off-diagonal elements are due to the Yukawa terms involving the Standard Model Higgs and introduce mixing between the electroweak singlets and doublets. We assume $Y_{l, e, \nu} v_{\ell} \gg y_{e, \nu} v, y_{e, \nu}^{\prime} v$, which is a phenomenologically natural assumption and frees the model from electroweak precision data constraints.

The mass eigenstates consist of six electrically neutral and six electrically charged states. As shown below, after $S U(2)_{\ell}$ breaking there remains a residual global $U(1)_{\ell}$ symmetry which prevents the new particles from decaying to solely Standard Model states. Therefore, if the lightest of the mass eigenstates is electrically neutral, it becomes a natural candidate for dark matter. This implies that the dark matter particle in the model is the $S U(2)_{\ell}$ partner of the right-handed neutrino, which after electroweak symmetry breaking receives a small admixture from the electroweak doublets:

$$
\begin{aligned}
& \chi_{L}=\nu_{L}^{\prime}+\epsilon \tilde{\nu}_{L}, \\
& \chi_{R}=\tilde{\nu}_{R}+\epsilon \nu_{R}^{\prime},
\end{aligned}
$$

where $\tilde{\nu}_{L}$ and $\nu_{R}^{\prime}$ are the upper components of the electroweak doublets $\tilde{l}_{L}$ and $l_{R}^{\prime}$, respectively, and $\epsilon \sim y_{\nu} v /\left(Y_{\nu} v_{\ell}\right) \sim y_{\nu}^{\prime} v /\left(Y_{\nu} v_{\ell}\right) \ll 1$.

The Higgs spectrum of the theory is that of a generic two Higgs doublet model. There are five physical scalar/pseudoscalar fields remaining after $S U(2)_{\ell}$ breaking. They are mixtures of the original CP-even and CP-odd components of $\hat{\Phi}_{1,2}$ and their masses depend on the choice of parameter values in the scalar potential (5).

Regarding the gauge sector, since there is no mixing between $S U(2)_{\ell}$ and the other gauge groups, after $S U(2)_{\ell}$ breaking the new vector gauge bosons develop equal masses,

$$
m_{Z^{\prime}, W_{ \pm}^{\prime}} \simeq \frac{1}{2} g_{\ell} v_{\ell}
$$

where $g_{\ell}$ is the $S U(2)_{\ell}$ gauge coupling. 


\begin{tabular}{|c||c||c|c|}
\hline Field & $U(1)_{\ell}$ & $U(1)_{L}$ & $U(1)_{\chi}$ \\
\hline \hline$\hat{l}_{L}$ & 1 & 1 & 0 \\
\hline$\hat{e}_{R}$ & 1 & 1 & 0 \\
\hline$\hat{\nu}_{R}$ & 1 & 0 & 1 \\
\hline \hline$l_{R}^{\prime}$ & 1 & 1 & 0 \\
\hline$e_{L}^{\prime}$ & 1 & 1 & 0 \\
\hline$\nu_{L}^{\prime}$ & 1 & 0 & 1 \\
\hline \hline$\Phi_{1,2}$ & 0 & 0 & 0 \\
\hline
\end{tabular}

Table 2: Charges under exact and approximate global symmetries.

\subsection{Global symmetries}

There exist two global symmetries of the Lagrangian. Only one of them, which we denote by $U(1)_{\ell}$, remains unbroken after $S U(2)_{\ell}$ breaking. Charges of the fields under this symmetry are provided in Table 2. Using the fact that the Yukawa couplings $y_{\nu}$ are tiny to account for the smallness of the Standard Model neutrino masses, and assuming that $y_{\nu}^{\prime}$ are small as well, the $U(1)_{\ell}$ global symmetry is promoted to two global symmetries, $U(1)_{L}$ and $U(1)_{\chi}$, which separately survive the breaking of $S U(2)_{\ell}$. The charges under those global symmetries are also shown in Table 2. Note that the charge under $U(1)_{L}$ can be interpreted as Standard Model lepton number, whereas the $U(1)_{\chi}$ charge is the dark matter number.

\section{$3 \quad$ Baryogenesis}

We now discuss the details of the baryon number asymmetry generation in the model. It relies on the fact that a primordial lepton asymmetry is produced by $S U(2)_{\ell}$ instantons The subsequent stages combine key features of several mechanisms: Dirac leptogenesis [20, 21], asymmetric dark matter [22, 23, 24, 25, 26, 27] and baryogenesis from an earlier phase transition [28].

${ }^{\dagger}$ We note that a similar idea was presented in Ref. [19], which was brought to our attention after the completion of this work. 


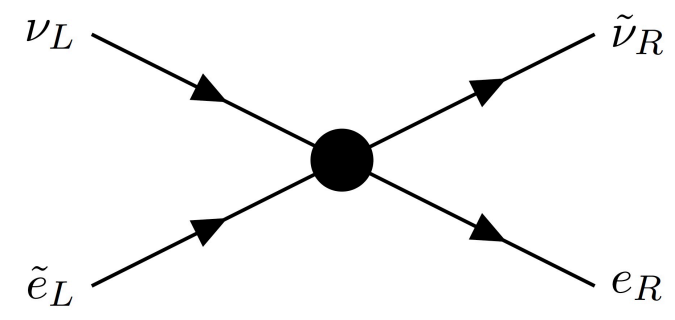

Figure 1: One of the interactions induced by $S U(2)_{\ell}$ instantons for which $\Delta L=$ -1 and $\Delta \chi=1$.

\section{1 $S U(2)_{\ell}$ instantons}

Because of the non-Abelian nature of $S U(2)_{\ell}$, the model exhibits nonperturbative dynamics in the form of $S U(2)_{\ell}$ instantons, which are active only above the $S U(2)_{\ell}$ breaking scale. The instantons preserve the global $U(1)_{\ell}$ symmetry, but they do not conserve the global $U(1)_{L}$ and $U(1)_{\chi}$ symmetries discussed in the previous section, since those symmetries are both anomalous under $S U(2)_{\ell}$ interactions. Following the calculation in Ref. [29], we find that the instantons induce the following dimension-six interaction terms:

$$
\begin{gathered}
\epsilon_{i j}\left[\left(l_{L}^{i} \cdot \bar{\nu}_{R}\right)\left(l_{L}^{j} \cdot \bar{e}_{R}\right)-\left(l_{L}^{i} \cdot \bar{\nu}_{R}\right)\left(\tilde{l}_{L}^{j} \cdot \overline{\tilde{e}}_{R}\right)+\left(l_{L}^{i} \cdot \tilde{l}_{L}^{j}\right)\left(\bar{\nu}_{R} \cdot \overline{\tilde{e}}_{R}\right)\right. \\
\left.-\left(l_{L}^{i} \cdot \tilde{l}_{L}^{j}\right)\left(\overline{\tilde{\nu}}_{R} \cdot \bar{e}_{R}\right)+\left(\tilde{l}_{L}^{i} \cdot \overline{\tilde{\nu}}_{R}\right)\left(\tilde{l}_{L}^{j} \cdot \overline{\tilde{e}}_{R}\right)-\left(\tilde{l}_{L}^{i} \cdot \overline{\tilde{\nu}}_{R}\right)\left(l_{L}^{j} \cdot \bar{e}_{R}\right)\right],
\end{gathered}
$$

where the dots denote Lorentz contractions and, for simplicity, we assumed just one generation of matter. The generalization to three families is straightforward.

The last term in (12), for example, generates two interaction terms, one of which gives rise to $\nu_{L} \tilde{e}_{L} \rightarrow \tilde{\nu}_{R} e_{R}$ shown in Fig. 1. For this process, as can be read off from Table 2, both the Standard Model lepton number and the dark matter number are violated by one unit: $\Delta L=-1$ and $\Delta \chi=1$, respectively. Therefore, the first condition for a successful leptogenesis, lepton number violation, is present in the model. 


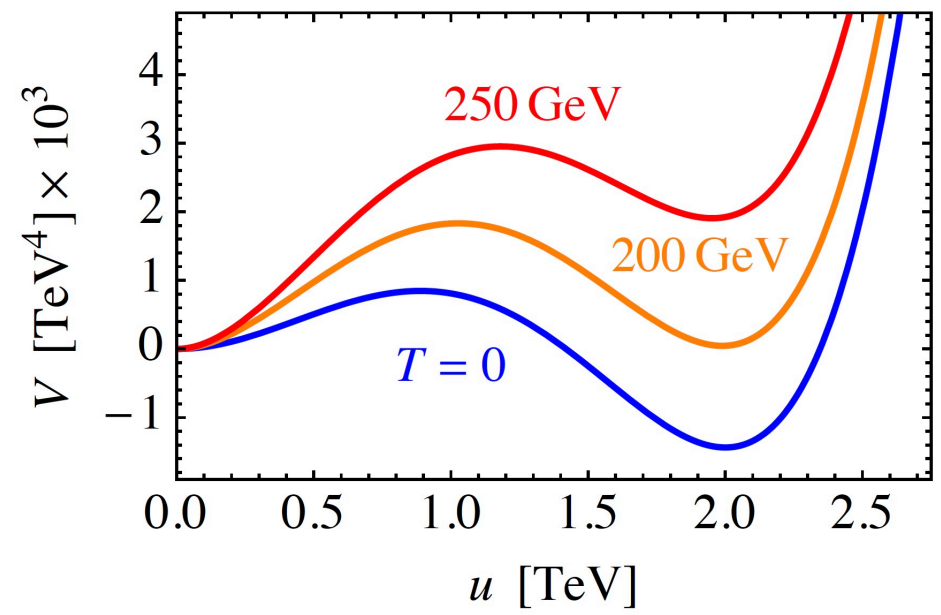

Figure 2: Plot of the finite temperature effective potential for $v_{\ell}=2 \mathrm{TeV}, \lambda_{1}=$ $2 \times 10^{-3}$ and $g_{\ell}=1$.

\subsection{CP violation and phase transition}

The remaining Sakharov conditions [30] require sufficient CP violation and out-of-equillibrium dynamics, which in our model can be realized via a first order phase transition. The scalar potential (5) contains four complex parameters: $m_{12}^{2}, \tilde{\lambda}_{5}, \tilde{\lambda}_{6}$, and $\tilde{\lambda}_{7}$. One phase can be rotated away by redefining the phase of $\hat{\Phi}_{1}^{\dagger} \hat{\Phi}_{2}$, leaving three physical phase combinations [31]. It is straightforward to show that for natural values of parameters the amount of $\mathrm{CP}$ violation in the model meets the criteria for a successful baryogenesis [1].

The last condition that needs to be checked is whether the model can actually accommodate a first order phase transition of the Universe. For this purpose we analyze the finite temperature effective potential [32], under the simplifying assumption $v_{1} \gg v_{2}$ :

$$
\begin{aligned}
V(u, T) & =-\frac{1}{2} m_{1}^{2} u^{2}+\frac{1}{4} \lambda_{1} u^{4} \\
& +\frac{1}{64 \pi^{2}} \sum_{i} n_{i}\left\{m_{i}^{4}(u)\left[\log \left(\frac{m_{i}^{2}(u)}{m_{i}^{2}\left(v_{\ell}\right)}\right)-\frac{3}{2}\right]+2 m_{i}^{2}(u) m_{i}^{2}\left(v_{\ell}\right)\right\} \\
& +\frac{T^{4}}{4 \pi^{2}} \sum_{i} n_{i} \int_{0}^{\infty} d x x^{2}\left[\log \left(1 \mp e^{-\sqrt{x^{2}+m_{i}^{2}(u) / T^{2}}}\right)-\log \left(1 \mp e^{-x}\right)\right]
\end{aligned}
$$




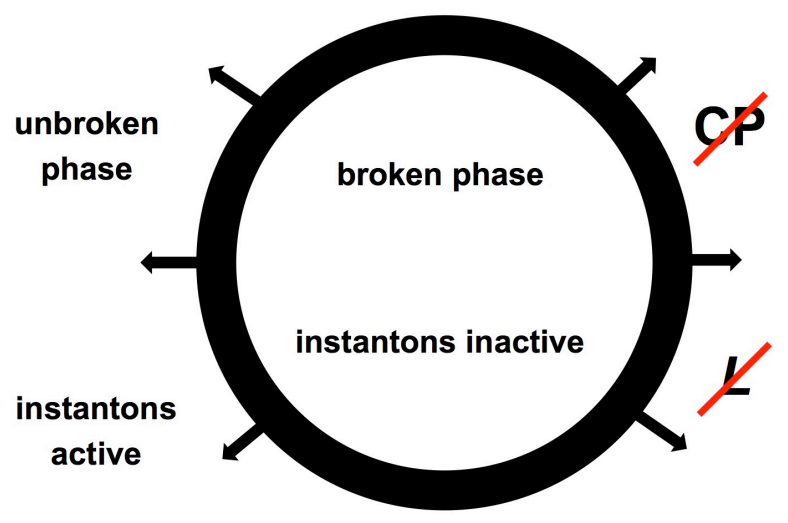

Figure 3: Expanding bubble of true vacuum.

where the first line is the tree-level Higgs contribution, the second line is the one loop zero temperature Coleman-Weinberg correction, and the last line is the finite temperature part. The sum is over all particles in the model, with appropriate factors corresponding to the number of degrees of freedom and statistics.

The plot of the effective potential is shown in Fig. 2 for a choice of parameters which sets the first order phase transition at the critical temperature $T_{c}=200 \mathrm{GeV}$. We chose $v_{\ell}=2 \mathrm{TeV}$, just above the current limit $v_{\ell} \gtrsim 1.7 \mathrm{TeV}$ set by the LEP-II experiment [15], so that the condition $v_{\ell}\left(T_{c}\right) / T_{c} \gtrsim 1$ for a strongly first order phase transition is fulfilled.

\subsection{Bubble nucleation and lepton asymmetry}

As the temperature of the Universe decreases and drops to $T_{c}$, bubbles of true vacuum start forming and expand, eventually filling out the entire Universe. A bubble expansion is schematically shown in Fig. 3. Outside the bubble the $S U(2)_{\ell}$ symmetry is not broken and the $S U(2)_{\ell}$ instantons remain active. Inside the bubble, on the other hand, $S U(2)_{\ell}$ is broken and the instanton effects are exponentially suppressed. As the bubble expands in the presence of $\mathrm{CP}$ violation, part of the lepton asymmetry generated by the instantons just outside the bubble becomes trapped inside the bubble. The same is true regarding the dark matter asymmetry. 
Although $S U(2)_{\ell}$ instantons are not active inside the bubble, one might worry whether the Standard Model lepton and dark matter asymmetries will be washed out by the Yukawa interactions involving $y_{\nu}$ and $y_{\nu}^{\prime}$, since they explicitly violate $U(1)_{L}$ and $U(1)_{\chi}$, while conserving only their sum $U(1)_{\ell}$. This, however, is not an issue, since the small values of $y_{\nu}$ and $y_{\nu}^{\prime}$ imply that the right-handed neutrinos and their partners reach chemical equillibrium long after the $S U(2)_{\ell}$ phase transition. As a result, the Standard Model lepton and dark matter number asymmetries survive until the electroweak phase transition, with just the lepton asymmetry being partially converted into a baryon asymmetry by the electroweak sphalerons, as discussed in the subsequent subsection.

The process of accumulation of the Standard Model lepton and dark matter asymmetries outside the expanding bubble is described by the diffusion equations [33, 34]:

$$
\dot{n}_{i}=D_{i} \nabla^{2} n_{i}-\sum_{j} \Gamma_{i j} \frac{n_{j}}{k_{j}}+\gamma_{i},
$$

where $n_{i}$ denotes the number density of a given particle species, $D_{i}$ is the diffusion constant, $\Gamma_{i j}$ is the diffusion rate, $k_{j}$ is the number of degrees of freedom times a factor arising from statistics and $\gamma_{i}$ is the $\mathrm{CP}$ violating source [35. In our model there is a set of twelve diffusion equations and eight constraints coming from the Yukawa and instanton interactions [1].

The solution to this set of diffusion equations is shown in Fig. 4, which plots the Standard Model lepton and dark matter particle number densities normalized to entropy as a function of the distance from the bubble wall located at $z=0$, where $z<0$ corresponds to the outside of the bubble and $z>0$ describes the inside of the bubble. The ratio of the generated Standard Model lepton and dark matter asymmetries in our model is:

$$
\left|\frac{\Delta L}{\Delta \chi}\right|=3
$$

and is independent of the model parameters. There exists a natural and experimentally allowed choice of parameters for which

$$
\frac{n_{L}}{s} \simeq 3 \times 10^{-10} .
$$




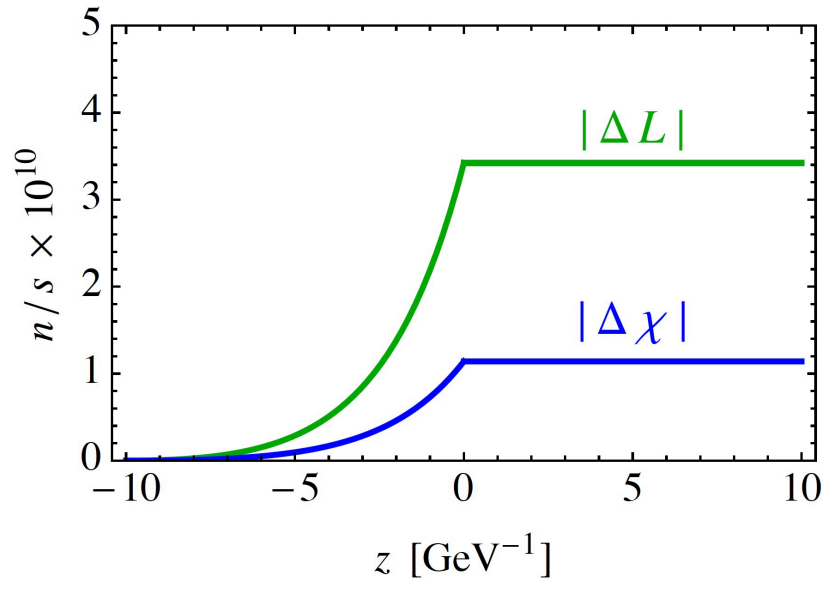

Figure 4: Standard Model lepton and dark matter particle number densities vs. distance from the bubble wall for a set of natural parameter values.

\subsection{Baryon asymmetry}

As mentioned earlier, the $S U(2)_{\ell}$ instantons become inactive after $S U(2)_{\ell}$ breaking and the dark matter number freezes in inside the bubble. This is not exactly the case for the Standard Model lepton asymmetry, since the Standard Model electroweak sphalerons remain active until the electroweak phase transition and convert part of the lepton number to baryon number. The resulting baryon asymmetry generated by the sphalerons is [36]

$$
\Delta B=\frac{28}{79} \Delta L
$$

therefore the final baryon asymmetry to entropy ratio is

$$
\frac{n_{B}}{s} \approx 10^{-10}
$$

which agrees with the observed value.

\section{Dark matter}

The dark matter candidate in our model 10 is composed mostly of the $S U(2)_{\ell}$ doublet partner of the right-handed neutrino. It is therefore predominantly a Standard Model singlet, with only a small admixture of an 

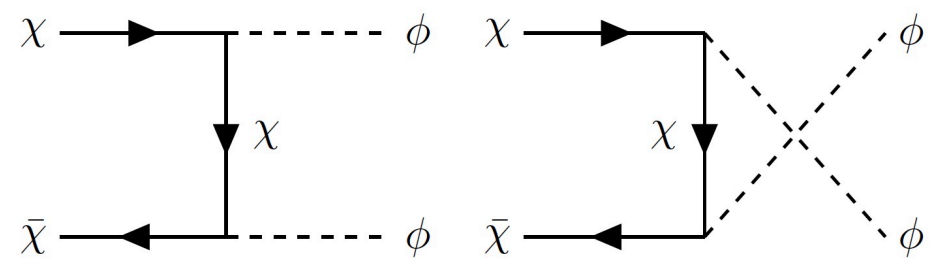

Figure 5: Dark matter annihilation to the pseudoscalar component of $\hat{\Phi}_{2}$.

electroweak doublet picked up by its interactions with the Standard Model Higgs field. Because the dark matter and baryon number asymmetries in our model are closely related (approximately equal at present time), the dark matter mass is uniquely determined by the observable ratio of the dark matter and baryonic relic densities. Assuming the dark matter is relativistic at the decoupling temperature, its mass is given by:

$$
m_{\chi}=m_{p} \frac{\Omega_{\mathrm{DM}}}{\Omega_{\mathrm{B}}}\left|\frac{\Delta B}{\Delta \chi}\right| \simeq 5 \mathrm{GeV} .
$$

A dark matter mass of a few $\mathrm{GeV}$ is generic in asymmetric dark matter models and it is generally challenging to make its symmetric component efficiently annihilate away. In the current model this issue is circumvented by arranging one of the Higgs components to be lighter than $5 \mathrm{GeV}$. In such a scenario a successful annihilation can proceed through the channels shown in Fig. 5. A light Higgs component can be realized provided that the quartic terms in the scalar potential are small, which in the case of $\lambda_{1}$ is also needed for a first order phase transition. This mass range for a new scalar/pesudoscalar is not strongly constrained by low energy experiments, but may be accessible in the future [37].

Since the new $Z^{\prime}$ gauge bosons do not interact with quarks, there are no tree-level direct detection diagrams involving the Standard Model singlet component of the dark matter. As a result, the direct detection constraint in this case can only arise from loop processes, but the GeV-scale dark matter limits coming from the CDMSlite experiment [38] are much less restrictive than the LEP-II constraint of $v_{\ell} \gtrsim 1.7 \mathrm{TeV}$, which we already took into account. Regarding the contribution of the direct detection diagrams involving the electroweak gauge bosons, it can be estimated using the results of Ref. [18] and is fully consistent with experiment in the phenomenologically natural limit $Y_{\nu} v_{\ell} \gg y_{\nu} v, y_{\nu}^{\prime} v$ we adopted. 


\section{Conclusions}

In this talk, I discussed a new model extending the Standard Model gauge group with a non-Abelian gauged lepton number $S U(2)_{\ell}$. The model realizes a mechanism for baryogenesis based on leptogenesis in which the lepton number asymmetry is generated by $S U(2)_{\ell}$ instantons. It also contains a natural dark matter candidate - the partner of the right-handed neutrino. Despite the theoretical advantages, it is difficult to test this theory experimentally. Since new physics resides in the lepton sector of the model, the best way to probe it would be in a new high energy lepton-lepton collider.

Let me end by saying that there is no reason not to expect the Standard Model gauge symmetry to be enhanced at higher energies. Analyzing other simple theories with extended gauge groups seems like a worthwhile effort, as it may shed more light on the outstanding issues of the Standard Model. However, one should always keep in mind that "Nature will do what Nature does and it's up to experiment to be the final judge'拥

\section{Acknowledgments}

I am grateful to Yuri Shirman, Tim Tait and Jennifer West for a fruitful collaboration. I would also like to thank the organizers of the Conference on Cosmology, Gravitational Waves and Particles in Singapore, especially the chairman, Harald Fritzsch, for the invitation, warm hospitality, and a fantastic scientific and social atmosphere during the conference. This research was supported in part by the DOE grant de-sc0009919 and the NSF grant PHY-1316792.

\section{References}

[1] B. Fornal, Y. Shirman, T. M. P. Tait, and J. R. West, "Asymmetric Dark Matter and Baryogenesis from SU(2)-Lepton," arXiv:1703.00199 [hep-ph], submitted to Phys. Rev. D.

[2] S. L. Glashow, "Partial Symmetries of Weak Interactions," Nucl. Phys. 22 (1961) 579-588

${ }^{\ddagger}$ From: M. B. Wise, interviewed by A. Ananthaswamy, article title: Hunt is on for quark dark matter, New Scientist, issue 3050, p. 10, published on December 5, 2015. 
[3] S. Weinberg, "A Model of Leptons," Phys. Rev. Lett. 19 (1967) 1264-1266.

[4] A. Salam, "Weak and Electromagnetic Interactions," Conf. Proc. C680519 (1968) 367-377.

[5] H. Fritzsch and M. Gell-Mann, "Current Algebra: Quarks and What Else?," Proceedings of the XVI International Conference on High Energy Physics, National Accelerator Laboratory, Chicago, p. 135165 (1972).

[6] H. Fritzsch, M. Gell-Mann, and H. Leutwyler, "Advantages of the Color Octet Gluon Picture," Phys. Lett. B47 (1973) 365-368.

[7] A. Pais, "Remark on Baryon Conservation," Phys. Rev. D8 (1973) 1844-1846.

[8] Y. Tosa, R. E. Marshak, and S. Okubo, "Baryon and Lepton Numbers as Broken Local Symmetries," Phys. Rev. D27 (1983) 444.

[9] S. Rajpoot, "Gauge Symmetries of Electroweak Interactions," Int. J. Theor. Phys. 27 (1988) 689.

[10] R. Foot, G. C. Joshi, and H. Lew, "Gauged Baryon and Lepton Numbers," Phys. Rev. D40 (1989) 2487-2489.

[11] C. D. Carone and H. Murayama, "Realistic Models with a Light U(1) Gauge Boson Coupled to Baryon Number," Phys. Rev. D52 (1995) 484-493, arXiv:hep-ph/9501220 [hep-ph].

[12] H. Georgi and S. L. Glashow, "Decays of a Leptophobic Gauge Boson," Phys. Lett. B387 (1996) 341-345, arXiv:hep-ph/9607202 [hep-ph].

[13] P. Fileviez Perez and M. B. Wise, "Baryon and Lepton Number as Local Gauge Symmetries," Phys. Rev. D82 (2010) 011901, arXiv:1002.1754 [hep-ph]. [Erratum: Phys. Rev.D82,079901(2010)].

[14] M. Duerr, P. Fileviez Perez, and M. B. Wise, "Gauge Theory for Baryon and Lepton Numbers with Leptoquarks," Phys. Rev. Lett. 110 (2013) 231801, arXiv:1304.0576 [hep-ph] 
[15] P. Schwaller, T. M. P. Tait, and R. Vega-Morales, "Dark Matter and Vectorlike Leptons from Gauged Lepton Number," Phys. Rev. D88 no. 3, (2013) 035001, arXiv:1305.1108 [hep-ph].

[16] J. M. Arnold, P. Fileviez Pérez, B. Fornal, and S. Spinner, "B and $L$ at the Supersymmetry Scale, Dark Matter, and R-Parity Violation," Phys. Rev. D88 no. 11, (2013) 115009, arXiv:1310.7052 [hep-ph].

[17] B. Fornal, A. Rajaraman, and T. M. P. Tait, "Baryon Number as the Fourth Color," Phys. Rev. D92 no. 5, (2015) 055022, arXiv:1506.06131 [hep-ph].

[18] B. Fornal and T. M. P. Tait, "Dark Matter from Unification of Color and Baryon Number," Phys. Rev. D93 no. 7, (2016) 075010, arXiv:1511.07380 [hep-ph].

[19] M. Blennow, B. Dasgupta, E. Fernandez-Martinez, and N. Rius, "Aidnogenesis via Leptogenesis and Dark Sphalerons," JHEP 03 (2011)

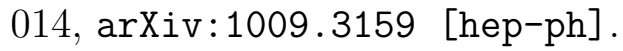

[20] K. Dick, M. Lindner, M. Ratz, and D. Wright, "Leptogenesis with Dirac Neutrinos," Phys. Rev. Lett. 84 (2000) 4039-4042, arXiv:hep-ph/9907562 [hep-ph].

[21] H. Murayama and A. Pierce, "Realistic Dirac Leptogenesis," Phys. Rev. Lett. 89 (2002) 271601, arXiv: hep-ph/0206177 [hep-ph]

[22] S. Nussinov, "Technocosmology? Could a Technibaryon Excess Provide a "Natural" Missing Mass Candidate?," Phys. Lett. B165 (1985) $55-58$.

[23] D. B. Kaplan, "A Single Explanation for Both the Baryon and Dark Matter Densities," Phys. Rev. Lett. 68 (1992) 741-743.

[24] D. Hooper, J. March-Russell, and S. M. West, "Asymmetric Sneutrino Dark Matter and the $\Omega_{b} / \Omega_{\mathrm{DM}}$ Puzzle," Phys. Lett. B605 (2005) 228-236, arXiv:hep-ph/0410114 [hep-ph].

[25] D. E. Kaplan, M. A. Luty, and K. M. Zurek, "Asymmetric Dark Matter," Phys. Rev. D79 (2009) 115016, arXiv:0901.4117 [hep-ph]. 
[26] K. Petraki and R. R. Volkas, "Review of Asymmetric Dark Matter," Int. J. Mod. Phys. A28 (2013) 1330028, arXiv:1305.4939 [hep-ph]

[27] K. M. Zurek, "Asymmetric Dark Matter: Theories, Signatures, and Constraints," Phys. Rept. 537 (2014) 91-121, arXiv:1308.0338 [hep-ph].

[28] J. Shu, T. M. P. Tait, and C. E. M. Wagner, "Baryogenesis from an Earlier Phase Transition," Phys. Rev. D75 (2007) 063510, arXiv:hep-ph/0610375 [hep-ph].

[29] D. E. Morrissey, T. M. P. Tait, and C. E. M. Wagner, "Proton Lifetime and Baryon Number Violating Signatures at the CERN LHC in Gauge Extended Models," Phys. Rev. D72 (2005) 095003, arXiv:hep-ph/0508123 [hep-ph].

[30] A. D. Sakharov, "Violation of CP Invariance, C Asymmetry, and Baryon Asymmetry of the Universe," Pisma Zh. Eksp. Teor. Fiz. 5 (1967) 32-35. [Usp. Fiz. Nauk161,61(1991)].

[31] J. F. Gunion and H. E. Haber, "Conditions for CP-Violation in the General Two-Higgs-Doublet Model," Phys. Rev. D72 (2005) 095002, arXiv:hep-ph/0506227 [hep-ph].

[32] M. Quiros, "Finite Temperature Field Theory and Phase Transitions," in High energy physics and cosmology. Proceedings, Summer School, Trieste, Italy, June 29-July 17, 1998, pp. 187-259. 1999. arXiv:hep-ph/9901312 [hep-ph].

[33] M. Joyce, T. Prokopec, and N. Turok, "Nonlocal Electroweak Baryogenesis. Part 1: Thin Wall Regime," Phys. Rev. D53 (1996) 2930-2957, arXiv:hep-ph/9410281 [hep-ph].

[34] A. G. Cohen, D. B. Kaplan, and A. E. Nelson, "Diffusion Enhances Spontaneous Electroweak Baryogenesis," Phys. Lett. B336 (1994) 41-47, arXiv:hep-ph/9406345 [hep-ph].

[35] A. Riotto, "Towards a Nonequilibrium Quantum Field Theory Approach to Electroweak Baryogenesis," Phys. Rev. D53 (1996) 5834-5841, arXiv:hep-ph/9510271 [hep-ph]. 
[36] J. A. Harvey and M. S. Turner, "Cosmological Baryon and Lepton Number in the Presence of Electroweak Fermion Number Violation," Phys. Rev. D42 (1990) 3344-3349.

[37] G. Krnjaic, "Probing Light Thermal Dark Matter With a Higgs Portal Mediator," Phys. Rev. D94 no. 7, (2016) 073009, arXiv:1512.04119 [hep-ph].

[38] SuperCDMS Collaboration, R. Agnese et al., "New Results from the Search for Low-Mass Weakly Interacting Massive Particles with the CDMS Low Ionization Threshold Experiment," Phys. Rev. Lett. 116 no. 7, (2016) 071301, arXiv:1509.02448 [astro-ph.C0] 